\title{
Brachial Flow-Mediated Dilation and Risk of Atrial Fibrillation in Older Adults: The Cardiovascular Health Study
}

This article was published in the following Dove Press journal: Vascular Health and Risk Management

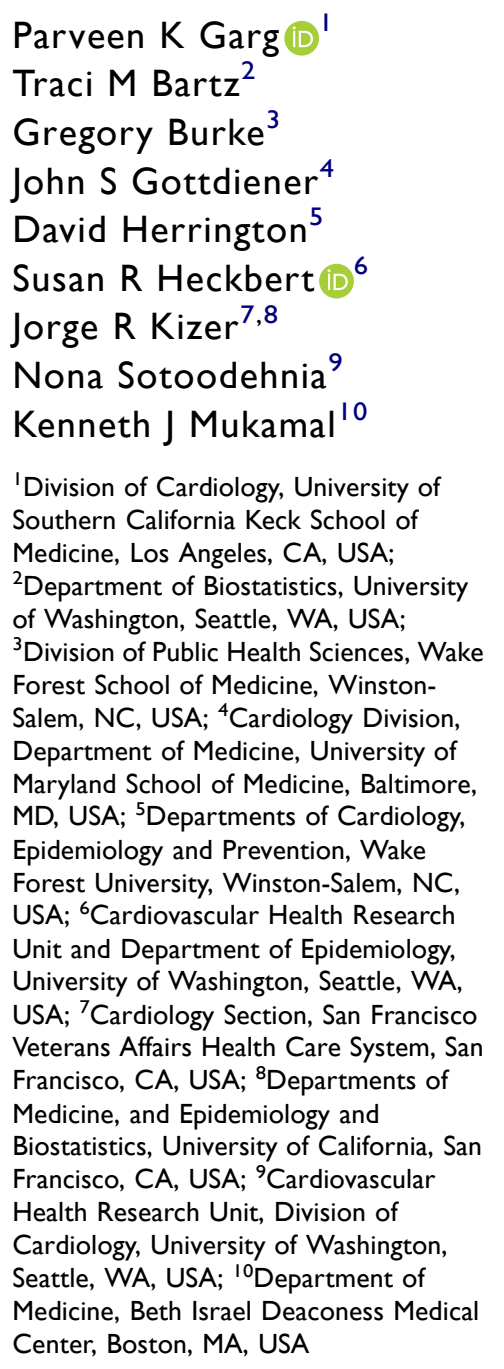

Correspondence: Parveen K Garg Division of Cardiology, University of Southern California Keck School of Medicine, 1510 San Pablo St. Suite 322, Los Angeles, CA, 90033, USA

Tel + 323-442-613|

Fax + I 323-442-6133

Email parveeng@med.usc.edu
Background: Endothelial dysfunction is associated with common risk factors for AF and has been implicated in the pathophysiology of atrial fibrillation (AF) through a variety of mechanisms. We determined the prospective association of brachial flow-mediated dilation (FMD) with incident AF among older adults.

Methods: We included 2027 Cardiovascular Health Study participants (mean age=78.3 years, male $=39 \%$, Black $=17 \%$ ) who underwent brachial FMD measurement at the 1997 to 1998 clinic visit. Incident AF was ascertained by study electrocardiograms, hospital discharge diagnosis coding and Medicare claims data. Cox regression models were used to examine the association between FMD and incident AF.

Results: We identified 754 incident of AF cases (37\%) over a median follow-up of 11.0 years. After adjusting for age, sex, race, height, weight, cardiovascular disease, cigarette smoking, hypertension, diabetes, kidney function, c-reactive protein, physical activity, alcohol consumption, and statins, the risk of AF did not differ according to brachial FMD response (4th vs 1 st quartile hazard ratio $(\mathrm{HR})=1.01,95 \%$ confidence interval (CI): 0.81 , 1.26; per FMD unit increment $\mathrm{HR}=1.01,95 \% \mathrm{CI}$ : 0.97, 1.05).

Conclusion: We found no relationship between brachial FMD and the risk of developing $\mathrm{AF}$ in this elderly cohort. Our findings suggest that the utility of brachial FMD as a risk marker for $\mathrm{AF}$ in older individuals is minimal.

Keywords: atrial fibrillation, arrhythmia, endothelium, flow-mediated dilation, subclinical disease

\section{Background}

Atrial fibrillation (AF) is the most commonly presenting cardiac arrhythmia in clinical practice. While only $2 \%$ of people younger than age 65 are estimated to have AF, both the prevalence and incidence of AF increase sharply after 65 years of age and more than $10 \%$ of patients aged at least 85 years have AF. ${ }^{1,2} \mathrm{~A}$ better understanding of AF disease mechanisms and identification of additional risk factors in older age are important.

Arterial flow-mediated dilation (FMD) is an indirect measure of endothelial nitric oxide (NO) release and, when impaired, is representative of underlying vascular endothelial dysfunction. ${ }^{3}$ Endothelial dysfunction is associated with common AF risk factors, including diabetes mellitus, hypertension, and smoking, presumably through mechanisms related to impaired NO release. ${ }^{4-7}$ In each of these studies, a significant difference was noted in the brachial FMD response for 
patients with each of these comorbidities compared to controls. In the case of smoking, a dose-related phenomenon was also observed in that total pack-years smoked was inversely related to brachial FMD response. ${ }^{6}$

Endothelial dysfunction has also been directly associated with the development of $\mathrm{AF}^{8,9}$ In an analysis of 2936 Multi-Ethnic Study of Atherosclerosis (MESA) participants, 137 of whom developed AF over a median follow-up period of 8.5 years, each standard deviation increase in brachial FMD response was associated with a $16 \%$ reduced risk of $\mathrm{AF}^{8}{ }^{8} \mathrm{~A}$ similar analysis was performed in the 3921 Framingham Heart Study participants, 270 of whom developed AF over a median follow-up period of 7.1 years. ${ }^{9}$ In this study, a $21 \%$ reduced risk of AF was observed with each standard deviation increase in brachial FMD response. ${ }^{9}$

Participants from both of these studies were relatively young (mean age $=61$ years) with a lower prevalence of important AF risk factors. Considering that older age is independently associated with a diminished brachial FMD response and that much of the AF risk is explained by older age and known risk factors, it is important to determine whether the relationship between brachial FMD and incident AF persists in an older population. ${ }^{10} \mathrm{We}$ determined the association of brachial FMD with the risk of developing $\mathrm{AF}$ in a population-based cohort of older adults.

\section{Methods}

\section{Study Participants}

The Cardiovascular Health Study (CHS) recruited 5888 participants in 2 waves (1989-1990 and 1992-1993) from four US field centers. ${ }^{11}$ In 1997-1998, 2791 of these participants underwent brachial artery ultrasound measurements. This was considered the baseline visit for the analysis. The study was approved by the institutional review board at each field center (Sacramento County, Sacramento, CA - University of California, Davis; Washington County, Hagerstown, MD - Johns Hopkins University; Forsyth County, Winston-Salem, NC - Wake Forest University School of Medicine; Pittsburgh, PA University of Pittsburgh) and was performed in accordance with the principles of the Declaration of Helsinki. All participants provided written informed consent.

We excluded 400 participants who had a history of AF at the time of measurement and another 195 participants with a pacemaker at baseline or who reported either digoxin or anti-arrhythmic drug use at any time prior to 1997-1998 due to the concern for suspected AF. We additionally excluded 169 participants who were missing either covariate or follow-up data.

\section{Brachial Flow-Mediated Dilation (FMD)}

The CHS scanning and reading protocol for brachial FMD has been previously published. ${ }^{12,13}$ Sonographers underwent centralized training in brachial FMD measurement at Wake Forest University School of Medicine and were deemed proficient after performing at least 20 acceptable scans on volunteers.

Participants were instructed to avoid caffeine, cigarettes or food for at least $8 \mathrm{~h}$ prior to the examination. All the examinations took place at approximately the same time (morning) in a room with an ambient temperature of 72 degrees Fahrenheit. After 15 minutes of rest in the fasting state, participants were examined in the supine position. The left arm was used for pulse and automated blood pressure monitoring at 5-minute intervals throughout the examination. A standard pediatric cuff was positioned around the right arm, $5 \mathrm{~cm}$ below the antecubital fossa. Right brachial artery images were captured using a 10-MHz Biosound Phase 2 ultrasound system (Biosound Esaote, Indianapolis, Ind). Baseline images of the right brachial artery were obtained for 2 minutes. Occlusion of the right brachial artery was then performed by inflating the pediatric cuff to $50 \mathrm{~mm} \mathrm{Hg}$ above the participant's systolic blood pressure and this was maintained for 4 minutes. Images of the right brachial artery were captured continuously for 2 minutes after cuff deflation. Images of the brachial artery diameters were captured in diastole (gated with ECG R wave). Videotapes of the acquired images of the brachial artery were analyzed at the Wake Forest University Cardiology Image Processing Laboratory with a previously validated semiautomated system. The semiautomated readings (media-adventitial interfaces to media-adventitial interfaces) of these digitized images generated the baseline and maximum diameters of the brachial artery from which \%FMD was computed. \%FMD was computed with the formula:

$$
\left[\frac{\text { maximum diameter - baseline diameter }}{\text { baseline diameter }}\right] \times 100 \%
$$

Correlations for repeated measures of baseline diameter, maximum diameter, and \%FMD were performed in 80 CHS participants scanned on 2 separate occasions more than 2 weeks apart and values were $0.94,0.94$, and 
0.67 , respectively. ${ }^{12}$ The reproducibility of the method was evaluated in 127 CHS participants based on two separate examinations less than 1 week apart. The mean \pm SD difference in percent change in diameter (brachial FMD) was $0.02 \pm 1.54 \%$, and $R^{2}$ was $0.7{ }^{14}$

\section{Atrial Fibrillation}

AF was identified from three sources: (1) ECGs obtained yearly at study examinations through 1998-1999 and interpreted by the EPICARE ECG reading center; ${ }^{15}$ (2) hospital discharge diagnoses with ICD-9 codes for AF or atrial flutter $(427.31,427.32)$ found through CHS hospitalization surveillance or Medicare claims data, excluding diagnoses assigned during the same hospitalization as coronary artery bypass or heart valve surgery, and (3) outpatient or physician service claims from Medicare data for AF or atrial flutter. AF was ascertained through December 2014. Participants were followed until the development of AF, death, or the end of the follow-up period, whichever came first.

\section{Covariates}

All variables were assessed at the time of FMD measurement, unless otherwise specified. Age, gender, race, smoking status (never, former, current), alcohol consumption, medication use (anti-hypertensive, including angiotensinconverting enzyme inhibitors and angiotensin receptor blockers, and statin use) and physical activity (kcals, measured 1996-1997) were obtained by self-report. Blood pressure, height (measured 1996-1997), and weight were directly measured by trained staff. C-reactive protein (CRP), fasting glucose, estimated glomerular filtration rate by cystatin C (eGFR), and NT-proBNP were all determined from serum samples taken in 1996-1997. Coronary heart disease (CHD), heart failure (HF), peripheral artery disease (PAD), and stroke were confirmed by physician adjudication.

\section{Statistical Analysis}

We described baseline characteristics across FMD response quartiles by mean (standard deviation) for continuous variables and $\mathrm{N}(\%)$ for categorical variables. We calculated $\mathrm{AF}$ incidence rates by FMD response quartiles. We used Cox regression models to examine the association between FMD (both as a continuous variable and by quartiles) and incident AF. In the first model, we adjusted for age, sex, race, and clinic site. In the second, we adjusted for the above and height, weight, CHD, HF, cigarette smoking, SBP, DBP, anti-hypertension medication use, diabetes, eGFR, CRP, physical activity, alcohol consumption, and statin use. We also repeated this analysis excluding participants either with baseline CVD (CHD, PAD, or stroke at the time of FMD measurement) both using the original models and additionally adjusting for incident CHD, PAD, and stroke during follow-up. Finally, because baseline brachial artery diameter was previously demonstrated to have the same prognostic accuracy as brachial FMD for CVD risk in this cohort, we repeated the above analysis to evaluate its relationship with AF as well. ${ }^{16}$ STATA version 12.1 was used for all analyses.

\section{Results}

A total of 2027 participants (mean age $=78.3$ years, male $=39 \%$, Black $=17 \%$ ) were included in the analysis and followed for a median duration of 11.0 years and a maximum duration of 17.5 years. Baseline characteristics according to brachial FMD response quartiles are shown in Table 1. We identified 754 incident cases of AF. Kaplan-Meier curves for the cumulative incidence of AF based on FMD response quartiles are shown in Figure 1. Compared to participants in the lowest quartile of brachial FMD, those in higher quartiles were not at a significantly reduced risk of incident AF (Table 2). Excluding participants with either baseline CVD did not meaningfully change the results (Table 3). Results were also similar when analyses were time-updated for incident CHD, PAD, or stroke during follow-up (Table 3). Baseline brachial artery diameter also showed no association with AF risk (adjusted hazard ratio per FMD unit increment $=0.93,95 \%$ confidence interval: $0.83,1.04$ ).

\section{Discussion}

We found no association of brachial FMD with the risk of incident AF amongst a population of older adults. Prior studies reporting a relationship between endothelial dysfunction and $\mathrm{AF}$ included substantially younger patient populations. $^{8,9}$

Endothelial dysfunction has previously been implicated in the pathophysiology of AF through a variety of mechanisms. Dysfunctional endothelium downregulates NO and upregulates adhesion molecules, leading to increased levels of inflammation and oxidative stress. NO has been shown to reduce electrical activity in cardiomyocytes isolated from the pulmonary vein and decreased levels may increase AF susceptibility. ${ }^{17}$ Increased inflammation can result in more atrial ectopy discharging from cells near the pulmonary veins. ${ }^{18,19}$ Finally, the generation of reactive 
Table I Baseline Characteristics of All CHS Participants According to Brachial FMD Response ( $n=2027)^{*}$

\begin{tabular}{|c|c|c|c|c|}
\hline \multirow[t]{3}{*}{ Characteristics } & \multicolumn{4}{|c|}{ Brachial FMD Percentage Change } \\
\hline & Quartile I & Quartile 2 & Quartile 3 & Quartile 4 \\
\hline & $(<1.9)$ & $(1.9-2.8)$ & $(2.9-4)$ & $(>4)$ \\
\hline Age, years (SD) & $78.6(4.5)$ & $78.6(4.5)$ & $78.2(4.2)$ & $78.0(4.0)$ \\
\hline Male, \% & $246(50.9)$ & $225(42.9)$ & $180(36.4)$ & $144(27.5)$ \\
\hline Black, \% & $125(25.9)$ & $99(18.9)$ & $68(13.7)$ & $59(11.3)$ \\
\hline Height, cm (SD) & $165(9.5)$ & $164(9.5)$ & $163(9.4)$ & $162(9.0)$ \\
\hline Weight, Ibs (SD) & $167(31.7)$ & $162(30.0)$ & $159(32.5)$ & $155(30.9)$ \\
\hline Systolic blood pressure, $\mathrm{mmHg}$ (SD) & $134(20.7)$ & $132(19.7)$ & $13 \mid(19.1)$ & $132(19.7)$ \\
\hline Diastolic blood pressure, $\mathrm{mmHg}(\mathrm{SD})$ & $67(10.7)$ & $68(10.3)$ & $66(10.8)$ & $67(10.0)$ \\
\hline Diabetes, \% & $90(18.6)$ & $91(17.3)$ & $82(16.6)$ & $76(14.5)$ \\
\hline eGFR, $\mathrm{mL} / \mathrm{min} / \mathrm{I} .73 \mathrm{~m}^{2}$ (SD) & $71.4(18.6)$ & $73.4(20.0)$ & $72.6(18.3)$ & $75.3(16.7)$ \\
\hline \multicolumn{5}{|l|}{ Smoking status, \% } \\
\hline Never & $183(37.9)$ & $212(40.4)$ & $204(4 I .2)$ & $206(39.3)$ \\
\hline Former & $266(55.1)$ & $278(53.0)$ & $250(50.5)$ & $28 I(53.6)$ \\
\hline Current & $34(7.0)$ & $35(6.7)$ & $4 \mid(8.3)$ & $37(7.1)$ \\
\hline Alcohol use, drinks/week (SD) & $2.3(5.7)$ & $2.1(5.4)$ & $2.2(6.6)$ & $2.2(6.1)$ \\
\hline Prevalent CHD, \% & $116(24.0)$ & $109(20.8)$ & $79(16.0)$ & $82(15.6)$ \\
\hline Prevalent PAD, \% & $13(2.7)$ & $10(1.9)$ & $12(2.4)$ & $10(1.9)$ \\
\hline Prevalent stroke, \% & $20(4.1)$ & $17(3.2)$ & $30(6.1)$ & $25(4.8)$ \\
\hline Heart failure, \% & $30(6.2)$ & $19(3.6)$ & $16(3.2)$ & $15(2.9)$ \\
\hline Anti-hypertensive use, $\%$ & $300(62.1)$ & $30 \mathrm{I}(57.3)$ & $278(56.2)$ & $243(46.4)$ \\
\hline ACEI use, \% & $89(18.4)$ & $64(12.2)$ & $66(13.3)$ & $47(9.0)$ \\
\hline ARB use, $\%$ & $10(2.1)$ & $8(1.5)$ & $15(3.0)$ & $10(1.9)$ \\
\hline Physical activity, kcals/week (SD) & $1445(1803)$ & |43| (1809) & $1315(1612)$ & $1349(1708)$ \\
\hline C-reactive protein, mg/dl (SD) & $3.9(4.5)$ & $4.9(8.7)$ & $4.7(9.0)$ & $4.4(6.3)$ \\
\hline Baseline brachial artery diameter, mm (SD) & $4.9(0.8)$ & $4.6(0.8)$ & $4.4(0.8)$ & $4.1(0.8)$ \\
\hline Percent FMD response & $1.2(0.5)$ & $2.4(0.3)$ & $3.4(0.3)$ & $5.7(1.5)$ \\
\hline Statin use, \% & $78(16.1)$ & $59(11.2)$ & $53(10.7)$ & $81(15.5)$ \\
\hline Incident $\mathrm{CHD}, \%^{\dagger}$ & $116(24.0)$ & $109(20.8)$ & $79(16.0)$ & $82(15.6)$ \\
\hline Incident PAD, \% ${ }^{\dagger}$ & $17(3.6)$ & $14(2.7)$ & $13(2.7)$ & $10(1.9)$ \\
\hline Incident stroke, $\%^{\dagger}$ & $4 \mid(8.9)$ & $51(10.0)$ & $33(7.1)$ & $46(9.2)$ \\
\hline
\end{tabular}

Notes: *Continuous variables are expressed as mean (SD). Categorical variables are $N$ (percent). †Participants with baseline CHD, PAD or stroke were not included in the percentage calculation.

Abbreviations: ACEI, angiotensin-converting enzyme inhibitor; ARB, angiotensin receptor blocker; CHD, coronary heart disease; FMD, flow-mediated dilation; eGFR, estimated glomerular filtration rate; PAD, peripheral artery disease. 


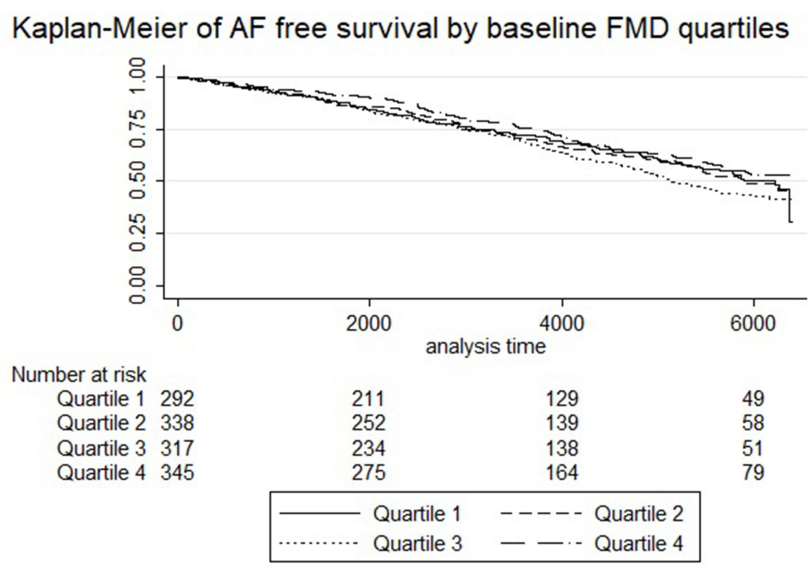

Figure I Kaplan-Meier of AF-free survival by baseline FMD quartiles. Abbreviations: AF, atrial fibrillation; FMD, flow-mediated dilation.

oxygen species and oxidative injury as a result of increased oxidative stress has been reported to precede the electrophysiological remodeling observed in $\mathrm{AF}^{20}$

Since much of the AF risk can be explained by age and clinical risk factors, it was important to determine whether the relationship between brachial FMD and incident AF persists in an older population. Results from two prior prospective population-based studies both report a significant inverse relationship between brachial FMD and incident AF risk. ${ }^{8,9}$ There are key differences in the study participant baseline characteristics between the current and prior studies, however, that may help to explain the discrepant findings. Participants from both of these prior studies were much younger (mean age of 61 years compared to 78 years). Additionally, participants from these earlier studies had a much lower prevalence of primary AF risk factors. The prevalence of antihypertensive medication use, diabetes, and smoking history in these studies were $29-34 \%, 9-13 \%$, and $46 \%$ (MESA study only) respectively. This compared to $55 \%$,
$18 \%$, and $60 \%$ in the current study. ${ }^{8,9}$ Finally, nearly $20 \%$ of CHS participants had established CHD compared to none in MESA and less than $5 \%$ in the Framingham Heart Study (only prevalent MI reported). Although coronary artery calcification was not available in CHS, it is likely that participants had a much higher subclinical atherosclerotic disease burden compared to those in earlier studies. Older age, traditional risk factors, and coronary artery disease are the strongest known risk factors for AF development. ${ }^{15,21-23}$ Considering the increased presence of each of these factors in our study population and their long-term impact on AF development, it is possible that the ability to evaluate the association of brachial FMD with AF in this setting was markedly reduced.

The average age of our study population being nearly 80 years old is important to recognize also because brachial FMD response diminishes with older age. ${ }^{23}$ In a small study brachial FMD response was 50-60\% lower in 60 to 79 years-old women compared with 20 to 30 years-old women $(8.1 \%$ vs $15.8 \%){ }^{24}$ Similarly, in a study of 36 healthy men, brachial FMD response was 30\% lower in middle-aged men compared to young men $(6.4 \%$ vs $8.7 \%){ }^{25}$ The utility of brachial FMD as a risk marker for AF in older individuals may be insignificant because the brachial FMD response is already poor by this time.

The degree of arterial stiffness that was likely present in the study participants may have also contributed to the lack of association seen here. The average diastolic blood and pulse pressures for the study participants were nearly identical $(\sim 65-70 \mathrm{mmHg})$, suggesting the presence of increased arterial stiffness, which is known to worsen with advancing age. A strong inverse correlation has been demonstrated between arterial stiffness measures and brachial FMD regardless of age or cardiovascular

Table 2 Association of Brachial FMD with Incident Atrial Fibrillation ( $n=2027)$

\begin{tabular}{|c|c|c|c|c|}
\hline & n/N (\%) AF/Total & Incidence Rate (/ 00 Person-Years) & $\begin{array}{c}\text { Model I* HR } \\
(95 \% \mathrm{Cl})\end{array}$ & $\begin{array}{c}\text { Model } 2^{\dagger} \mathbf{H R} \\
(95 \% \mathrm{Cl})\end{array}$ \\
\hline \multicolumn{5}{|l|}{$\%$ FMD } \\
\hline $\mathrm{I}^{\text {st }}$ quartile $(<1.9)$ & $17 \mid / 483(35.4)$ & 3.91 & I (referent) & I (referent) \\
\hline $2^{\text {nd }}$ quartile $(1.9-2.8)$ & $190 / 525(36.2)$ & 4.01 & $0.97(0.79,1.20)$ & $\mathrm{I} .00(0.8 \mathrm{I}, \mathrm{I} .23)$ \\
\hline $3^{\text {rd }}$ quartile $(2.9-4)$ & 205/495 (4I.4) & 4.50 & $1.09(0.89,1.35)$ & $1.15(0.93,1.42)$ \\
\hline $4^{\text {th }}$ quartile $(>4)$ & I88/524 (35.9) & 3.78 & $0.92(0.74,1.14)$ & I.0I $(0.8 \mathrm{I}, \mathrm{I} .26)$ \\
\hline Per FMD unit increment & $754 / 2027$ (37.2) & & $0.99(0.95,1.03)$ & $1.01(0.97,1.05)$ \\
\hline
\end{tabular}

Notes: *Model I adjusted for age, sex, race, and clinic site. ${ }^{\dagger}$ Model 2 adjusted for Model I + height, weight, coronary heart disease, congestive heart failure, cigarette smoking, SBP, DBP, use of anti-hypertensive medication, statin use, diabetes, eGFR, CRP, physical activity, and alcohol consumption.

Abbreviations: AF, atrial fibrillation; FMD, flow-mediated dilation; $\mathrm{HR}$, hazard ratio; $\mathrm{Cl}$, confidence interval. 
Table 3 Association of Brachial FMD with Incident Atrial Fibrillation in Individuals without Baseline Cardiovascular Disease ( $\mathrm{n}=1557)$

\begin{tabular}{|c|c|c|c|c|c|}
\hline & n/N (\%) AF/Total & $\begin{array}{l}\text { Incidence Rate } \\
\text { (/100 Person-Years) }\end{array}$ & $\begin{array}{l}\text { Model I* HR } \\
(95 \% \mathrm{Cl})\end{array}$ & $\begin{array}{l}\text { Model } 2^{\dagger} \text { HR } \\
(95 \% \mathrm{Cl})\end{array}$ & Model $3^{t \dagger}$ \\
\hline$\%$ FMD & & & & & \\
\hline$I^{\text {st }}$ quartile $(<1.9)$ & $121 / 353(34.3)$ & 3.63 & I (referent) & I (referent) & I (referent) \\
\hline $2^{\text {nd }}$ quartile $(1.9-2.8)$ & $142 / 404(35.1)$ & 3.72 & $0.98(0.77,1.26)$ & $1.01(0.79,1.30)$ & $1.01(0.87,1.17)$ \\
\hline $3^{\text {rd }}$ quartile $(2.9-4)$ & I60/387 (4I.3) & 4.33 & $1.15(0.90,1.47)$ & $1.17(0.92,1.50)$ & $1.08(0.93,1.26)$ \\
\hline $4^{\text {th }}$ quartile $(>4)$ & $143 / 4 \mid 6(34.4)$ & 3.43 & $0.91(0.71,1.16)$ & $0.96(0.74,1.23)$ & $0.92(0.79,1.06)$ \\
\hline Per FMD unit increment & $566 / 1560(36.3)$ & & $0.99(0.95,1.04)$ & $1.01(0.96,1.05)$ & $0.98(0.96,1.01)$ \\
\hline
\end{tabular}

Notes: *Model I adjusted for age, sex, race, and clinic site. ${ }^{\dagger}$ Model 2 adjusted for Model I + height, weight, coronary heart disease, congestive heart failure, cigarette smoking, SBP, DBP, use of anti-hypertensive medication, statin use, diabetes, eGFR, CRP, physical activity, and alcohol consumption. ${ }^{\dagger \dagger}$ Model 3 adjusted for Model $2+$ interim development of incident CHD, PAD, and stroke.

Abbreviations: AF, atrial fibrillation; FMD, flow-mediated dilation; $\mathrm{HR}$, hazard ratio; $\mathrm{Cl}$, confidence interval.

disease status. ${ }^{26-28}$ In one such study of 262 never-treated hypertensive patients between 30 and 55 years of age, each $\mathrm{mmHg}$ increment in pulse pressure was associated with an $8.7 \%$ decrease in FMD response. ${ }^{26}$ The reduction in shear stress seen with elevations in pulse pressure may be a primary contributing factor to FMD impairment by increasing oxidative stress and reducing the production of nitric oxide. ${ }^{26}$ Therefore, the level of arterial stiffness already present may have played a role in the reduced range of FMD response seen in this population.

Our study has limitations. Our method for AF detection was not able to distinguish amongst AF subtypes and the overall sensitivity for capturing cases of paroxysmal AF, especially those that are asymptomatic, was likely reduced. The study population was comprised of older, predominantly white individuals and findings cannot be generalized to younger and/or non-white populations. The duration or severity of adjusted risk factors was not available. Arterial stiffness measures were not available in this cohort and correlations with brachial FMD response could not be performed. Individuals with chronic inflammation such as autoimmune diseases, infectious diseases or active cancer were not excluded; however, doing so would likely only move results more towards the null. Finally, certain baseline covariates were measured from an earlier clinic visit.

\section{Conclusion}

In conclusion, we found no relationship between brachial FMD and the risk of developing AF in this older cohort.

\section{Abbreviations}

$\mathrm{AF}$, atrial fibrillation; CHD, coronary heart disease; CHS, Cardiovascular Health Study; CRP, c-reactive protein; CVD, cardiovascular disease; eGFR, estimated glomerular filtration rate; FMD, flow-mediated dilation; HF, heart failure; MESA, Multi-Ethnic Study of Atherosclerosis; NO, nitric oxide; PAD, peripheral artery disease.

\section{Ethics Approval and Consent to Participate}

The study was approved by the institutional review board at each center, and all participants provided written informed consent.

\section{Author Contributions}

All authors made substantial contributions to the content and writing of this paper. PKG and KJM conceptualized the study. TMB did the statistical analysis. JSD, JRK, SRH, PKG, KJM provided feedback on the analysis and results. PKG wrote the original draft. All authors contributed to data analysis, drafting or revising the article, have agreed on the journal to which the article will be submitted, gave final approval of the version to be published, and agree to be accountable for all aspects of the work.

\section{Funding}

This research was supported by contracts HHSN 268201200036C, HHSN268200800007C, HHSN2682 01800001C, N01HC55222, N01HC85079, N01HC85080, N01HC85081, N01HC85082, N01HC85083, N01HC85086, and grants U01HL080295 and U01HL130114 from the National Heart, Lung, and Blood Institute (NHLBI), with additional contribution from the National Institute of Neurological Disorders and Stroke (NINDS). Additional support was provided by R01AG023629 from the National Institute on Aging (NIA). A full list of principal CHS investigators and institutions can be found at CHS-NHLBI.org. 


\section{Disclosure}

The authors take responsibility for all aspects of the reliability and freedom from bias of the data presented and their discussed interpretation.

Ms Traci M Bartz and Dr Susan R Heckbert report grants from NIH, during the conduct of the study. Dr Jorge R Kizer reports stock ownership in Bristol Myers Squibb, Johnson \& Johnson, Medtronic, Merck, and Pfizer, outside the submitted work. The authors declare that they have no other potential conflicts of interest for this work.

\section{References}

1. Boriani G, Diemberger I, Martignani C, Biffi M, Branzi A. The epidemiological burden of atrial fibrillation: a challenge for clinicians and health care systems. Eur Heart J. 2006;27(8):893-894. doi:10.1093/eurheartj/ehi651

2. Andrade J, Khairy P, Dobrev D, Nattel S. The clinical profile and pathophysiology of atrial fibrillation: relationships among clinical features, epidemiology, and mechanisms. Circ Res. 2014;114 (9):1453-1468. doi:10.1161/CIRCRESAHA.114.303211

3. Celermajer DS, Sorensen KE, Spiegelhalter DJ, Georgakopoulos D, Robinson J, Deanfield JE. Aging is associated with endothelial dysfunction in healthy men years before the age-related decline in women. J Am Coll Cardiol. 1994;24(2):471-476. doi:10.1016/07351097(94)90305-0

4. McVeigh GE, Brennan GM, Johnston GD, et al. Impaired endothelium-dependent and independent vasodilation in patients with type 2 (non-insulin-dependent) diabetes mellitus. Diabetologia. 1992;35(8):771-776. doi:10.1007/BF00429099

5. Paniagua OA, Bryant MB, Panza JA. Role of endothelial nitric oxide in shear stress-induced vasodilation of human microvasculature: diminished activity in hypertensive and hypercholesterolemic patients. Circulation. 2001;103(13):1752-1758. doi:10.1161/01. CIR.103.13.1752

6. Celermajer DS, Sorensen KE, Georgakopoulos D, et al. Cigarette smoking is associated with dose-related and potentially reversible impairment of endothelium-dependent dilation in healthy young adults. Circulation. 1993;88(5):2149-2155. doi:10.1161/01.CIR.88.5.2149

7. Weiner SD, Ahmed HN, Jin Z, et al. Systemic inflammation and brachial artery endothelial function in the Multi-Ethnic Study of Atherosclerosis (MESA). Heart. 2014;100(11):862-866 doi:10.1136/heartjnl-2013-304893

8. O'Neal WT, Efird JT, Yeboah J, et al. Brachial flow-mediated dilation and incident atrial fibrillation: the multi-ethnic study of atherosclerosis. Arterioscler Thromb Vasc Biol. 2014;34 (12):2717-2720. doi:10.1161/ATVBAHA.114.304560

9. Shaikh AY, Wang N, Yin X, et al. Relations of arterial stiffness and brachial flow-mediated dilation with new-onset atrial fibrillation: the Framingham Heart Study. Hypertension. 2016;68(3):590-596. doi:10.1161/HYPERTENSIONAHA.116.07650

10. Celermajer DS, Sorensen KE, Gooch VM, et al. Non-invasive detection of endothelial dysfunction in children and adults at risk of atherosclerosis. Lancet. 1992;340(8828):1111-1115. doi:10.1016/ 0140-6736(92)93147-F

11. Fried LP, Borhani NO, Enright P, et al. The Cardiovascular Health Study: design and rationale. Ann Epidemiol. 1991;1(3):263-276. doi:10.1016/1047-2797(91)90005-W
12. Herrington DM, Fan L, Drum M, et al. Brachial flow-mediated vasodilator responses in population-based research: methods, reproducibility and effects of age, gender and baseline diameter. J Cardiovasc Risk. 2001;8(5):319-328. doi:10.1177/174182670100800512

13. Garg PK, Tan AX, Odden MC, et al. Brachial Flow-mediated Dilation and Risk of Dementia. Alzheimer Dis Assoc Disord. 2020;34(3):272-274. doi:10.1097/WAD.0000000000000394

14. Herrington DM, Espeland MA, Crouse JR, et al. Estrogen replacement and brachial artery flow-mediated vasodilation in older women. Arterioscler Thromb Vasc Biol. 2001;21(12):1955-1961. doi:10.1161/ hq1201.100241

15. Psaty BM, Manolio TA, Kuller LH, et al. Incidence of and risk factors for atrial fibrillation in older adults. Circulation. 1997;96 (7):2455-2461. doi:10.1161/01.CIR.96.7.2455

16. Yeboah J, Crouse JR, Hsu F, Burke GL, Herrington DM. Brachial flow-mediated dilation predicts incident cardiovascular events in older adults: the Cardiovascular Health Study. Circulation. 2007;115 (18):2390-2397. doi:10.1161/CIRCULATIONAHA.106.678276

17. Lin YK, Lu YY, Chen YC, Chen YJ, Chen SA. Nitroprusside modulates pulmonary vein arrhythmogenic activity. J Biomed Sci. 2010;17(1):20. doi:10.1186/1423-0127-17-20

18. Haissaguerre M, Jais P, Shah DC, et al. Spontaneous initiation of atrial fibrillation by ectopic beats originating in the pulmonary veins. $N$ Engl J Med. 1998;339(10):659-666. doi:10.1056/NEJM19980 9033391003

19. Aviles RJ, Martin DO, Apperson-Hansen C, et al. Inflammation as a risk factor for atrial fibrillation. Circulation. 2003;108 (24):3006-3010. doi:10.1161/01.CIR.0000103131.70301.4F

20. Kim YM, Guzik TJ, Zhang YH, et al. A myocardial Nox2 containing $\mathrm{NAD}(\mathrm{P}) \mathrm{H}$ oxidase contributes to oxidative stress in human atrial fibrillation. Circ Res. 2005;97(7):629-636. doi:10.1161/01. RES.0000183735.09871.61

21. Benjamin EJ, Levy D, Vaziri SM, D’Agostino RB, Belanger AJ, Wolf PA. Independent risk factors for atrial fibrillation in a population-based cohort: the Framingham Heart Study. JAMA. 1994;271(11):840-844. doi:10.1001/jama.1994.03510350050036

22. Garg PK, O'Neal WT, Chen LY, et al. American heart association's life simple 7 and risk of atrial fibrillation in a population without known cardiovascular disease: the ARIC (Atherosclerosis Risk in Communities) Study. J Am Heart Assoc. 2018;7(8):e008424. doi:10.1161/JAHA.117.008424

23. Heeringa J, van der Kuip DA, Hofman A, et al. Subclinical atherosclerosis and risk of atrial fibrillation: the Rotterdam Study. Arch Intern Med. 2007;167(4):382-387. doi:10.1001/archinte.167.4.382

24. Parker BA, Ridout SJ, Procter DN. Age and flow-mediated dilation: a comparison of dilatory responsiveness in the brachial and popliteal arteries. Am J Physiol Heart Circ Physiol. 2006;291(6):H3043H3049. doi:10.1152/ajpheart.00190.2006

25. Nakamura A, Kajitani S, Sato K, et al. Decline of popliteal artery flow-mediated dilation with aging and possible involvement of asymmetric dimethylarginine in healthy men. $J$ Med Ultrason. 2001;46 (4):503-511. doi:10.1007/s10396-019-00946-2

26. Ceravolo R, Maio R, Pujia A, et al. Pulse pressure and endothelial dysfunction in never-treated hypertensive patients. $J$ Am Coll Cardiol. 2003;41(10):1753-1758. doi:10.1016/S0735-1097(03)00295-X

27. Beigel R, Dvir D, Arbel Y, Shechter A, Feinberg MS, Shechter M. Pulse pressure is a predictor of vascular endothelial function in middle-aged subjects with no apparent heart disease. Vasc Med. 2010;15(4):299-305. doi:10.1177/1358863X10373300

28. Soga J, Nakamura S, Nishioka K, et al. Relationship between augmentation index and flow-mediated vasodilation in the brachial artery. Hypertens Res. 2008;31(7):1293-1298. doi:10.1291/ hypres.31.1293 


\section{Publish your work in this journal}

Vascular Health and Risk Management is an international, peerreviewed journal of therapeutics and risk management, focusing on concise rapid reporting of clinical studies on the processes involved in the maintenance of vascular health; the monitoring, prevention and treatment of vascular disease and its sequelae; and the involvement of metabolic disorders, particularly diabetes. This journal is indexed on PubMed Central and MedLine. The manuscript management system is completely online and includes a very quick and fair peerreview system, which is all easy to use. Visit http://www.dovepress com/testimonials.php to read real quotes from published authors. 